\title{
BMJ Open Protocol for a prospective cohort study: Prevention of Transmissions by Effective Colonisation Tracking in Neonates (PROTECT-Neo)
}

\author{
Tim Götting (D) , ${ }^{1}$ Sandra Reuter, ${ }^{1}$ Daniel Jonas, ${ }^{1}$ Roland Hentschel, ${ }^{2}$ \\ Philipp Henneke, ${ }^{2,3}$ Daniel Klotz, ${ }^{2}$ Simone Hock, ${ }^{2}$ Martin Wolkewitz, ${ }^{4}$ \\ Benjamin Blümel, ${ }^{5}$ Georg Häcker, ${ }^{5}$ Hajo Grundmann, ${ }^{1}$ Nico Mutters (D) ${ }^{1,6}$
}

To cite: Götting T, Reuter S, Jonas D, et al. Protocol for a prospective cohort study: Prevention of Transmissions by Effective Colonisation Tracking in Neonates (PROTECT-Neo). BMJ Open 2020;10:e034068. doi:10.1136/ bmjopen-2019-034068

- Prepublication history for this paper is available online. To view these files, please visit the journal online (http://dx.doi. org/10.1136/bmjopen-2019034068).

Received 04 September 2019 Revised 19 August 2020 Accepted 19 August 2020

D) Check for updates

(c) Author(s) (or their employer(s)) 2020. Re-use permitted under CC BY-NC. No commercial re-use. See rights and permissions. Published by BMJ.

For numbered affiliations see end of article.

\section{Correspondence to} Dr Tim Götting; tim.goetting@uniklinik-freiburg. de

\section{ABSTRACT}

Introduction Transmissions of opportunistic bacterial pathogens between neonates increase the risk of infections with negative repercussions, including higher mortality, morbidity and permanent disabilities. The probability of transmissions between patients is contingent on a set of intrinsic (patient-related) and extrinsic (wardrelated) risk factors that are not clearly quantified. It is the dual objective of the Prevention of Transmissions by Effective Colonisation Tracking-Neo study to determine the density of transmission events in a level III neonatal intensive care unit (NICU) and to identify risk factors that may be causally associated with transmission events. Methods and analysis A full cohort of patients treated in a 17-bed level III NICU will be prospectively followed and transmission events between two or more patients will be documented. A transmission event occurs when isogenic isolates from two different patients can be identified. Isolates will be obtained by routine weekly screening. Isogenicity will be determined by whole-genome sequencing. During the study, relevant intrinsic and extrinsic risk factors will be recorded. Specimen and data will be collected for 1 year. We postulate that transmission density increases during episodes when demand for intensive care cannot be met by existing staff, and that threshold dynamics have a bearing on cohorting and hand hygiene performance. Poisson logistic regression, proportional hazard and multilevel competing risk models will be used to estimate the effect of explanatory variables. Ethics and dissemination This study has been approved by the local ethics committee (study ID 287/18). The results will be published in peer-reviewed medical journals, communicated to participants, the general public and all relevant stakeholders.

Trial registration number The German Clinical Trials Registry (DRKS00017733); Pre-results.

\section{INTRODUCTION}

For various preventive purposes related either to maternal (in case of caesarean section) or child health (colonisation with group B streptococcus), neonates are exposed to antibiotics in up to $30 \%$ of cases, even in the
Strengths and limitations of this study

- Continuous screening of a complete level III neonatal intensive care unit (NICU) cohort, as well as wholegenome sequencing and typing of the most common nosocomial pathogens, will allow ascertainment of nosocomial transmission dynamics in neonates.

- Prospective enrolment of the total population at risk (all premature neonates), recording of risk factors/ confounders and analysis using appropriate multilevel competing risk models will increase understanding of transmission dynamics in the NICU.

- The single-centre study design may limit the ability to generalise findings in other settings.

- The study results may form the basis for designing multicentre intervention trials for tailored measures to prevent transmission events.

absence of individual signs of infection. Moreover, preterm infants are frequently treated with antibiotics immediately after birth, since clinical signs of infection are non-specific and common in this patient group. ${ }^{1}$ Neonate vulnerability to the acquisition of nosocomial, often antibiotic resistant, pathogens during treatment in neonatal intensive care units (NICU) is related to an immature immune system, because neonates and especially those delivered by caesarean section have no protective microbiome, and are hence only sparsely colonised by bacteria. ${ }^{1-4}$

Accordingly, NICU house a unique cohort of exquisitely susceptible patients, who, being colonised by particular nascent microbiome communities, readily serve as recipients of opportunistic pathogens through transmission from others patients, healthcare workers or the hospital environment. ${ }^{15}$ Since the transmitted pathogens originate from nosocomial sources rather than from the maternal microbiome, they are likely more tenacious and 
antibiotic resistant, and, consequently, also challenging to treat when causing infections. Hence, transmissions in NICUs often occur in clusters and cause outbreaks of infection, with dire consequences in terms of mortality, morbidity and permanent disabilities in patients, economic consequences, as well as possibly damaging the treating hospital's reputation. ${ }^{67}$ Data on colonisation dynamics in neonates are scarce and the impact of pathogens appearing in microbiological samples is difficult to assess. Whether colonisation with facultative pathogenic bacteria represents 'natural' microbiota assembled from contact with the parents, or whether they rather result from horizontal transmissions between NICU patients remains in most cases unresolved. However, bacteria transmitted horizontally-in contrast to bacteria transmitted vertically-that is, natural microbiome transfer from parents to offspring, are presumably more virulent, more frequently drug-resistant or multidrug-resistant and have a higher tenacity, since these bacteria often originate from hospital-adapted nosocomial lineages. The only way to shed more light on this issue is by rigorous molecular typing of all bacterial isolates within a defined patient cohort in an NICU setting.

Furthermore, other variables or risk factors for transmission of pathogens, in many cases, the prerequisite for infection, are poorly investigated in NICU patients. The impact of compliance with infection control measures, especially hand hygiene, also needs to be analysed. Other important factors that may influence transmission, such as bed occupancy rates, staffing levels (patient-nurse ratio per work shift) and the amount of nursing care a patient requires, must also be considered.

\section{Aims and objectives}

To discriminate between natural colonisation and transmissions, we have designed a study on the frequency of transmission of nosocomial pathogens in an NICU setting. During the study, we will screen for the most relevant nosocomial pathogens for NICU patients (Enterobacterales, Acinetobacter baumannii, Pseudomonas aeruginosa, Staphylococcus aureus), many of which are not only among the earliest colonising bacteria, but are also often associated with severe infections.

The main aim of the study is the identification of transmission events by (1) complete typing of all selected nosocomial pathogens using amplified fragment-length polymorphism (AFLP) and (2) whole-genome sequencing (WGS) of all strains that are not distinguishable by AFLP. Since we will use WGS, this will also allow for an identification of the so-called 'high-risk clones' and will allow analysis of evolution and transmission of circulating lineages and their resistance genes. ${ }^{8}$

The secondary aim of the study is the assessment of relevant risk factors that may influence transmission dynamics. Our primary hypothesis is that transmission events in the NICU occur more often when negative extrinsic risk factors accumulate (eg, during times of high bed occupancy, unfavourable nurse to patient ratio, neonates with extreme intensive care demand and high colonisation pressure which means a considerable number of patients colonised with the same pathogen at the time of a transmission event). Our secondary hypothesis is that horizontal transmissions between patients lead to subsequent infections more frequently than vertical transmission. Therefore infection densities will be compared for patients who are part of one or more transmission events (cases) and those who are not involved in any transmission event (controls).

In an approach to control for confounding further patient-related variables will be collected and analysed. Thus distribution of variables such as date of birth, birth weight, gestational age as well as exposure to kangaroo care, procedures, diagnostic measures and consultation visits will be examined.

\section{METHODS AND ANALYSIS}

\section{Study site}

The single-centre prospective cohort study will be conducted at the NICU of the Medical Center-University of Freiburg, one of the largest tertiary care hospitals in Germany (with 2000 beds). The NICU has 17 beds, which consist of 8 fully equipped ICU beds and 9 intermediate care beds, and provides level III neonatal care (definition according to the American Academy of Pediatrics). ${ }^{9}$

Around 350 term and preterm newborns (20\% of these with a birth weight of less than $1500 \mathrm{~g}$ ), are admitted annually to the NICU. This unit covers the entire spectrum of diseases and congenital malformations in the preterm and term newborn infant. Accordingly, the NICU meets the highest standards of current neonatal care. It can, therefore, be assumed that patients at this NICU represent a cross-section of all the patients receiving care at maximum level neonatal units in Germany and possibly in other countries with highly developed healthcare systems, too.

\section{Study population}

Inclusion criteria: All patients admitted for $\geq 48$ hours who have been screened at least once will be included in the study.

Exclusion criteria: All patients with a stay of $<48$ hours, and all patients who have not been screened will be excluded.

\section{Microbiological methods}

Weekly screening of all NICU patients will be carried out in accordance with the national guidelines of the RobertKoch Institute, Berlin, Germany. ${ }^{10}$ The screening regimen will include nasopharyngeal, rectal and, if applicable, wound or stoma swabs (eSwab, Copan, Brescia, Italy). Screening swabs and clinical samples will be inoculated on suitable media and incubated under aerobic conditions for 48 hours at $36^{\circ} \mathrm{C}$ and with $\mathrm{CO}_{2} 5 \%$. If growth on plates is detected, identification of microorganisms will be performed by matrix-assisted laser desorption 
ionisation-time-of-flight mass spectrometry (Bruker Daltonics, Bremen, Germany). Susceptibility testing will be performed using VITEK2 (Biomérieux, Nuertingen, Germany) or MIC test strips (Liofilchem, Piane Romano, Italy), respectively, and the results will be interpreted according to EUCAST (European Committee on Antimicrobial Susceptibility Testing) clinical breakpoints. Presence of resistance genes will be confirmed by nucleic acid amplification tests for methicillin-resistant $S$. aureus and carbapenem-resistant Gram negatives.

\section{Sample collection}

Both wild-type and antimicrobial resistant variants of bacterial pathogens obtained from screening and clinical specimens will be collected and kept at $-80^{\circ} \mathrm{C}$ for the duration of the study. The following indicator pathogens will be included: $S$. aureus, Acinetobacter calcoaceticus $-A$. baumannii complex and Enterobacterales.

\section{Typing methods}

The molecular genotyping procedure is stepwise. (I) Identification and susceptibility testing of isolated pathogens; (II) genotyping by AFLP will be applied as previously described. ${ }^{11}$ S. aureus isolates will undergo spa typing as described elsewhere ${ }^{12}$; (III) clonal lines, defined as frequently occurring strains of the same species that cannot be differentiated by AFLP or spa typing will be further analysed using WGS to identify high-risk clones. WGS will be performed with the Illumina Nextera DNA Flex Kit library preparation and $2 \times 150$ bp paired-end reads. Incidence of transmissions (defined as transmission of a pathogen from one patient to the other as assessed by molecular typing) will be calculated per 1000 patient days.

\section{Outcomes}

\section{Primary outcome}

Transmission events: pathogens of the same genus/species in different patients which are indistinguishable after molecular typing.

\section{Secondary outcomes}

Incidence of multidrug-resistant and susceptible indicator pathogens: according to EUCAST clinical breakpoints; standard quantitative statistics.

Infection rates: density (infections/patient days $\times 1000$ )

\section{Collection of influencing risk factors, possible confounders and further variables}

Throughout the study period the following risk factors, confounders and variables potentially influencing transmission dynamics will be extracted prospectively from patient charts or electronic systems (laboratory information system, clinical information system) by a study nurse and an infection control nurse (table 1):

To generate an estimate of compliance with hand hygiene throughout the study period regular observations of hand hygiene performance will be conducted on a monthly basis following an established protocol (measured as number of hand disinfections/number of valid indications $\times 100){ }^{13}$

\section{Statistical considerations}

Data will be collected at individual patient as well as aggregated (monthly and unit) level. In a time series, analysis-approach time periods during which transmission events occur will be compared with those without transmission events in terms of the risk factors (namely bed occupancy rate, patient to nurse ratio, care efforts

Table 1 Risk factors, confounders and further variables

\begin{tabular}{|c|c|}
\hline Risk factors & Measuring method/unit \\
\hline Bed occupancy rate & Utilisation (occupancy days/possible occupancy days×100) \\
\hline Patient to nurse ratio & Number of patients/day/number of nurses/day \\
\hline Care efforts & INPULS (Intensivpflege und Leistungserfassungssystem) care categories ${ }^{16}$ \\
\hline colonisation pressure & Number of patients colonised with the same pathogen concordant to place and time ${ }^{17}$ \\
\hline Confounders/further variables & Measuring method/unit \\
\hline Standard patient characteristics & Date of birth, birth weight, gestational weight \\
\hline Localisation of the patient & Bed place; ID of the incubator \\
\hline Kangaroo care & Received or not received for each patient \\
\hline Antibiotic consumption & $\begin{array}{l}\text { Application density (number of treatment days/number of patient days } \times 1000 \text { per } \\
\text { antibiotic substance); overall consumption measured in DDDs (defined daily dose) per } \\
\text { antibiotic substance/class }\end{array}$ \\
\hline Device use & Number of catheter days/number of patient days $\times 100$ per catheter type \\
\hline Ventilation days & $\begin{array}{l}\text { Number of ventilation days/number of patients days } \times 100 \text { per non-invasive and invasive } \\
\text { ventilation types, respectively }\end{array}$ \\
\hline Surgical procedures & Type and number of procedure/patient \\
\hline Invasive diagnostic measures & Type and number of measure/patient \\
\hline Consultation visits & Type and number/patient \\
\hline
\end{tabular}


and colonisation pressure). Furthermore, patients who are part of a transmission event (donor or recipient of a bacterial pathogen) and patients who are not part of a transmission event will be compared and evaluation of possible confounding considering further variables will be performed. Analysis will include univariate and multivariate approaches. For univariate analysis, rate ratios (RRs), 95\% CIs and $p$ values will be determined for categorical exposure variables. Association of the dependent with continuous variables (eg, gestational age, body weight, days of relative staff deficit, etc) will be tested for significant differences by score test for trend. RRs will be reported as estimates for one unit increase in exposure. Poisson or negative binomial appropriate regression models will be used for multivariate analysis and precautions will be taken to avoid overfitting. Stringent criteria and stepwise entry into a forward approach will optimise then numbers of candidate variables. Effect modification will be tested by fitting interaction terms into the regression. All data will be collected into an MS Access database and analysed using the statistical software SAS 7.4, STATA and $\mathrm{R}$. The primary analysis regarding the transmission events will be performed on monthly and unit level. We plan to include 100-140 patients. As 350 mostly preterm newborns are admitted annually to the NICU sample size will be achieved in the given time. All outcomes for the included patients and corresponding isolates will be analysed. We expect 50-70 transmissions per year (about five per month). A sample size calculation for the Poisson distribution ${ }^{14}$ yields that with an observation period of 12 months, we can detect a group difference between 3.5 and 6 transmissions per months. After examining the distribution (Poisson, negative binomial), appropriate regression models will be adapted to determine the transmission rate as a function of factors (bed occupancy rate, patient to nurse ratio, care efforts and colonisation pressure).$^{15}$ If applicable, we will use the patient-individual level data for secondary analyses.

\section{Patient and public involvement}

No patients were involved in the design of the study nor its conduction. The public will be informed on publication of the study. Publication will be in an open-access manner.

\section{DISCUSSION}

We expect our results to form the foundation of informing infection prevention and control (IPC) strategies in the neonatal setting. We believe that effective transmission tracking and assessing relevant confounders as well as other possible influencing variables will improve patient safety. We aim to identify so-called control points that can be used in subsequent multicentre studies to implement randomised controlled IPC interventions.

\section{ETHICS AND DISSEMINATION}

No specific invasive measures will be taken or medication applied within the scope of our planned study. In other words, all the patients will receive standard medical care that is not influenced by the study. Furthermore, no patient will be denied therapy or preventive measures or additional diagnostics, whether included or excluded from the study. The results of the microbiological samples and the resistance patterns of isolated bacteria will be available to the treating physicians as usual, and will possibly influence patient care, for example, empiric antibiotic therapy. All additional patient data collected exclusively for this study will be kept inaccessible to third parties and analysed in anonymous form. No identifiable patient data will be published or permanently stored. Parental consent is not required as collection and analysis of the microbiological isolates is purely descriptive and corresponds to infection control quality criteria according to the German Infection Protection Act (IfSG) and the KRINKO (Kommission für Krankenhaushygiene und Infektionsprävention) commission as outlined in $\$ 23$ section 1 IfSG. According to and in compliance with the WMA (World Medical Association) Declaration of Helsinki, Helsinki, Finland, June 1964, last revised in October 2013 in Fortaleza, Brazil, there are in our opinion no ethical concerns regarding the conduct of the study. The study was approved by the Ethics Committee, Medical Centre-University of Freiburg, on 28 August 2018 (registration number 287/18).

The results of the study should contribute to the development of targeted infection-preventive measures and adapted antibiotic therapy, thereby increasing patient safety for the extremely vulnerable study population of neonates. The results will be presented at national and international scientific meetings. Additionally, we aim to publish the results, preferably in open-access journals, to guarantee broad access for interested audiences. The sequencing data will be made available by uploading them to common online platforms.

\section{Author affiliations}

${ }^{1}$ Institute for Infection Prevention and Hospital Epidemiology, Medical CenterUniversity of Freiburg, Faculty of Medicine, University of Freiburg, Freiburg, Germany ${ }^{2}$ Center for Pediatrics and Adolescent Medicine, Medical Center-University of Freiburg, Faculty of Medicine, University of Freiburg, Freiburg, Germany ${ }^{3}$ Institute for Immunodeficiency (CCI), Medical Center-University of Freiburg, Faculty of Medicine, University of Freiburg, Freiburg, Germany ${ }^{4}$ Institute of Medical Biometry and Statistics, Medical Center-University of Freiburg, Faculty of Medicine, University of Freiburg, Freiburg, Germany ${ }^{5}$ Institute of Medical Microbiology and Hygiene, Medical Center-University of Freiburg, Faculty of Medicine, University of Freiburg, Freiburg, Germany ${ }^{6}$ Institute for Hygiene and Public Health, University Hospital Bonn, Bonn, Germany

Acknowledgements The authors would like to thank the whole team on the neonatal ward for their continued support and help throughout the planning of the study. The authors especially would like to thank Hildegard Wolf from the Institute for Infection Prevention and Hospital Epidemiology and NICU nurse Rebecca Leukart for all their work and effort. The authors also thank Deborah Lawrie-Blum for language editing.

Contributors TG and NTM are the principal investigators of the study. They initiated, designed and drafted the protocol and coordinated all collaborations, 
wrote the publication and reviewed the intellectual content of this paper. $\mathrm{HG}$ advised on designing the study. HG, DJ and SR advised on the sequencing methodology and on the phylogenetic analysis, and reviewed this paper for intellectual content. RH, PH, DK and SH provided clinical advice and made critical revisions. MW advised on the statistical design of the study and reviewed this paper for intellectual content. BB and GH advised on sampling strategies for microbiological samples and reviewed this paper for intellectual content. All listed authors have and will participate in the study, and have reviewed and approved the final manuscript.

Funding This study is being funded by the programme 'Clinical Studies' of the Medical Faculty of the Albert-Ludwig University of Freiburg, Freiburg, Germany.

Competing interests None declared.

Patient and public involvement Patients and/or the public were not involved in the design, or conduct, or reporting, or dissemination plans of this research.

Patient consent for publication Not required.

Provenance and peer review Not commissioned; externally peer reviewed.

Open access This is an open access article distributed in accordance with the Creative Commons Attribution Non Commercial (CC BY-NC 4.0) license, which permits others to distribute, remix, adapt, build upon this work non-commercially, and license their derivative works on different terms, provided the original work is properly cited, appropriate credit is given, any changes made indicated, and the use is non-commercial. See: http://creativecommons.org/licenses/by-nc/4.0/.

\section{ORCID iDs}

Tim Götting http://orcid.org/0000-0002-7753-3361

Nico Mutters http://orcid.org/0000-0002-0156-9595

\section{REFERENCES}

1 Brooks B, Olm MR, Firek BA, et al. The developing premature infant gut microbiome is a major factor shaping the microbiome of neonatal intensive care unit rooms. Microbiome 2018;6:112.

2 Raveh-Sadka T, Thomas BC, Singh A, et al. Gut bacteria are rarely shared by co-hospitalized premature infants, regardless of necrotizing enterocolitis development. Elife 2015:4:1-3.

3 Groer MW, Luciano AA, Dishaw LJ, et al. Development of the preterm infant gut microbiome: a research priority. Microbiome 2014;2:38.
4 Freudenhammer M, Henneke P, Härtel C. Mikrobiom von Risikoneugeborenen und präventive Modifikation. Monatsschr Kinderheilkd 2019;167:411-9.

5 Maródi L. Neonatal innate immunity to infectious agents. Infect Immun 2006;74:1999-2006.

6 Stone PW, Gupta A, Loughrey M, et al. Attributable costs and length of stay of an extended-spectrum beta-lactamase-producing Klebsiella pneumoniae outbreak in a neonatal intensive care unit. Infect Control Hosp Epidemiol 2003;24:601-6.

7 Kadambari S, Botgros A, Clarke P, et al. Characterizing the burden of invasive Pseudomonas infection on neonatal units in the UK between 2005 and 2011. J Hosp Infect 2014;88:109-12.

8 Aanensen DM, Feil EJ, Holden MTG, et al. Whole-Genome sequencing for routine pathogen surveillance in public health: a population snapshot of invasive Staphylococcus aureus in Europe. mBio 2016;7:1.

9 American Academy of pediatrics Committee on fetus and newborn. levels of neonatal care. Pediatrics 2012;130:587-97.

10 Empfehlung R-K-IE. zur „Prävention nosokomialer Infektionen bei neonatologischen Intensivpflegepatienten mit einem Geburtsgewicht unter 1.500 g" (2007). Epidemiologisches Bulletin 2011;2012:13-15.

11 Jonas D, Spitzmüller B, Daschner FD, et al. Discrimination of Klebsiella pneumoniae and Klebsiella oxytoca phylogenetic groups and other Klebsiella species by use of amplified fragment length polymorphism. Res Microbiol 2004;155:17-23.

12 Harmsen D, Claus H, Witte W, et al. Typing of methicillin-resistant Staphylococcus aureus in a university hospital setting by using novel software for spa repeat determination and database management. $J$ Clin Microbiol 2003;41:5442-8.

13 Sax H, Allegranzi B, Chraïti M-N, et al. The world Health organization hand hygiene observation method. Am J Infect Control 2009;37:827-34.

14 Belt V. G statistical rules of thumb. 2nd edn. Hoboken, NJ: John Wiley \& Sons, 2008.

15 Wolkewitz M, Cooper BS, Palomar-Martinez M, et al. Multilevel competing risk models to evaluate the risk of nosocomial infection. Crit Care 2014;18:R64.

16 Joa C, Faschingbauer C. [Calculating cost effectiveness in intensive care nursing: INPULS (Intensive Nursing and and Performance System) clarifies expenditures]. Pflege Z 2003;56:651-5.

17 Bonten MJ, Slaughter S, Ambergen AW, et al. The role of "colonization pressure" in the spread of vancomycin-resistant enterococci: an important infection control variable. Arch Intern Med 1998;158:1127-32. 